\title{
Surface plasmon resonance-induced photoactivation of gold nanoparticles as bactericidal agents against methicillin- resistant Staphylococcus aureus
}

\author{
This article was published in the following Dove Press journal: \\ International Journal of Nanomedicine \\ 22 March 2014 \\ Number of times this article has been viewed
}

\section{Lucian Mocan' \\ loana llie ${ }^{2}$ \\ Cristian Matea' \\ Flaviu Tabaran' \\ Ersjebet Kalman' \\ Cornel lancu' \\ Teodora Mocan ${ }^{3}$ \\ '3rd Surgery Clinic, Department of Nanomedicine, "Iuliu Hatieganu" University of Medicine and Pharmacy, Cluj-Napoca, Romania; '2Department of Endocrinology, Department of Nanomedicine, "Iuliu Hatieganu" University of Medicine and Pharmacy, Cluj-Napoca, Romania; ${ }^{3}$ Department of Physiology, Department of Nanomedicine, "luliu Hatieganu" University of Medicine and Pharmacy, Cluj-Napoca, Romania}

\begin{abstract}
Systemic infections caused by methicillin-resistant Staphylococcus aureus (MRSA) and other bacteria are responsible for millions of deaths worldwide, and much of this mortality is due to the rise of antibiotic-resistant organisms as a result of natural selection. Gold nanoparticles synthesized using the standard wet chemical procedure were photoexcited using an $808 \mathrm{~nm}$ $2 \mathrm{~W}$ laser diode and further administered to MRSA bacteria. Flow cytometry, transmission electron microscopy, contrast phase microscopy, and fluorescence microscopy combined with immunochemical staining were used to examine the interaction of the photoexcited gold nanoparticles with MRSA bacteria. We show here that phonon-phonon interactions following laser photoexcitation of gold nanoparticles exhibit increased MRSA necrotic rates at low concentrations and short incubation times compared with MRSA treated with gold nanoparticles alone. These unique data may represent a step forward in the study of bactericidal effects of various nanomaterials, with applications in biology and medicine.
\end{abstract}

Keywords: MRSA, SPR, multi-drug resistant bacteria, infection, gold nanoparticles, laser

\section{Introduction}

Resistance to antimicrobial drugs represents a looming and increasingly serious public health crisis. ${ }^{1}$ Systemic infections caused by methicillin-resistant Staphylococcus aureus (MRSA) and other bacteria are responsible for millions of deaths worldwide, and much of this mortality is due to the rise of antibiotic-resistant organisms as a result of natural selection. Of particular concern are hospitalized patients, presenting an increased risk of contracting bacterial diseases during their hospital stay each year. Because antibiotic-resistant infections double the duration of hospital stay, as well as mortality and morbidity rates, when compared with drug-susceptible infections, extensive stewardship campaigns have been promoted to encourage prudent use of antibiotics and to limit unnecessary exposure to these compounds. ${ }^{2}$

MRSA isolates are resistant to all available penicillins and other $\beta$-lactam antimicrobial drugs and represent a dangerous pathogen for both community-acquired as well as hospital-associated infections. ${ }^{3}$ MRSA has emerged as a significant nosocomial pathogen in the last decade and is now endemic in most European countries. ${ }^{4}$ This bacterium is a major public health danger because its eradication from hospitals is very difficult as it exhibits genetic variation in bacterial populations. ${ }^{5}$ Thus, individual bacteria develop several mutations that make antibiotics ineffective, resulting in the proliferation of the resistance trait. ${ }^{6}$
Correspondence: Teodora Mocan Department of Physiology, Department of Nanomedicine, "luliu Hatieganu" University of Medicine and Pharmacy, 8 Victor Babes St, 4000I 2 Cluj-Napoca, Romania

Email teodora.mocan@umfcluj.ro 
Although new antibiotics are currently being developed, these represent different versions of the existing drug classes for which MRSA exhibit decreased susceptibility. Newer antibiotics may help to offset the onward march of MRSA resistance, but it has become increasingly apparent that new technologies for rapid detection of MRSA carriage and treatment are needed. ${ }^{7}$

Nanotechnology might be crucial for drug delivery, and nanoparticles are of high importance in the optimization of drug delivery to target sites in the body. These nanoparticles can potentially be engineered as drug-delivery tools. ${ }^{8}$

During the last decade, advances in functionalization chemistry have been one of the driving forces in the development of new classes of novel nanomaterials for applications in biology and medicine. ${ }^{9-15}$ Gold nanoparticles (GNPs) with their physiochemical malleability and large surface area can strongly absorb near-infrared (NIR) radiance in the visible region by inducing coherent collective oscillation of conduction band electrons in strong resonance with visible frequencies of light. ${ }^{16}$

This phenomenon is often termed surface plasmon resonance (SPR), and it is one of the most explored characteristics of this noble metal. ${ }^{17,18}$ During laser excitation, the resonance frequency of SPR is strongly dependent upon the size, shape, interparticle interactions, as well as on the dielectric constant of the surrounding medium. ${ }^{19}$ Thus, when nanoparticle size increases, the collective oscillation of the conduction electrons located on its surface is translated into SPR red-shifts. ${ }^{20}$

The photothermal properties of GNPs have been systematically studied by several authors using femtosecond transient absorption spectroscopy, showing that laser photoexcited gold nanostructures yield exceptionally high absorption coefficients and scattering properties within the ultraviolet (UV)-visible wavelength range, ${ }^{14,21,22}$ allowing their applicability in nanophotothermolysis experiments..$^{20,23,24}$

This SPR response as a consequence of laser irradiation is followed by phonon-phonon interactions, where the nanoparticle lattice cools rapidly by exchanging energy within a layer of the medium surrounding the nanoparticles, for instance cell or bacteria. ${ }^{18,25,26}$

The aim of our research was to study whether pretreatment NIR activation of GNPs enhances the toxicity of nanoparticles in MRSA organisms. We hypothesized that laser photoexcitation of the conduction electrons which oscillate at the surface of GNPs (prior to administration to MRSA bacteria) enhance the nonradiative relaxation through electron-phonon and subsequent phonon-phonon coupling mechanisms, leading to bacterial membrane disruption. This is, to our knowledge, the first report that describes the increased necrotic efficacy in anti-MRSA treatment following administration of plasmonic GNPs.

\section{Methods}

\section{Reagents}

Reagents needed for the synthesis of GNPs were purchased from Sigma-Aldrich (St Louis, MO, USA). The immunoassay kits (LIVE/DEAD ${ }^{\circledR}$ BacLight ${ }^{\mathrm{TM}}$ Bacterial Viability test) used in the experiments were purchased from Life Technologies (Carlsbad, CA, USA).

\section{Synthesis of GNPs}

GNPs were obtained using $10 \mathrm{mg}$ sodium borohydride dissolved in $120 \mathrm{~mL}$ of deionized water, further added to $50 \mathrm{~mL}$ of $200 \mu \mathrm{mol} / \mathrm{L}$ aqueous solution of tetracholoroauric acid. The mixture underwent continuous stirring for 24 hours. The obtained product was further centrifuged at $10,000 \times \mathrm{g}$ for 45 minutes at $10^{\circ} \mathrm{C}$. The resulting GNPs were filtered through a $0.22-\mu \mathrm{m}$ filter and used as described elsewhere. ${ }^{27}$

\section{Characterization of GNPs}

A Shimadzu UV-Mini 1240 (Shimadzu Corporation, Kyoto, Japan) UV-visible spectrophotometer was used to analyze the characteristic SPR band of the GNPs. ${ }^{28,29}$ The size of the nanoparticles was assessed using Zetasizer DLS (Malvern Instruments, Malvern, UK) system.

\section{Nanoparticle therapeutics}

MRSA bacteria in our experiments were cultured in amino acid-rich aqueous solutions (Sigma-Aldrich) until reaching a flow cytometric density of $5-6 \times 10^{3} / \mathrm{mL}$ (in $\mathrm{O}_{2}$ atmosphere). The plasmon resonance-induced NIR-activated GNPs were added to the MRSA solution and incubated at various time intervals ( 1 minute, 30 minutes, 1, 5, and 24 hours) and at increasing concentrations (1, 5, 20, and 50 $\mathrm{mg} / \mathrm{L})$. Following incubation, the MRSA-containing tubes were placed in a high speed ultracentrifuge at $11,000 \times g$ and centrifuged for 10 minutes. The resulting pellet was further re-immersed into a clean solution of amino acids. Every experiment was performed in triplicate for all concentrations.

\section{Culture and characterization of MRSA}

A swab sample intraoperatively collected from a surgical patient after an intra-abdominal abscess was directly inoculated into an MRSA selective agar and further incubated 
(for 48 hours at $35^{\circ} \mathrm{C}$ in $\mathrm{O}_{2}$ atmosphere). The MRSA colony was confirmed by tube coagulase and DNase testing. Resistance to MRSA was assessed using cefoxitin disk screen test. Multidrug-resistant MRSA bacteria were defined as microorganisms possessing resistance to all nonB-lactam antibiotics, including tetracycline, erythromycin, ciprofloxacin, and trimethoprim/sulfamethoxazole.

The minimum inhibitory concentration (MIC) method was used to evaluate bactericidal effect of both types of GNPs. The MIC classic methodology (dilution tests) proposed by Mazzola et $\mathrm{al}^{30}$ was adapted to 96 -well microplates as described by dos Santos et al. ${ }^{7}$

The bacteria concentration we used to assess antimicrobial effectiveness of GNPs/pre-GNPs was approximately $10^{4} \mathrm{CFU} / \mathrm{mL}$. The minimum inhibitory concentration was considered the percentage of the original GNP/pre-GNP dispersion in the medium. Thus, an MIC value of $50 \%$ indicates a solution containing $50 \%$ of the nanoparticles in the original synthesis dispersion. To minimize the evaporation of the culture medium of the test wells during the incubation, $200 \mu \mathrm{L}$ of deionized sterile water was added on the inner perimeter wells of the microplates. One hundred microliters of the culture medium was distributed in 12 of the 96 wells, except for well 1. In the first and second wells of the microplate row, $100 \mu \mathrm{L}$ of the GNP dispersion was added. Both wells were mixed with a micropipette, and $100 \mu \mathrm{L}$ was next transferred from well 2 to well 3 and successively repeated until well 11 . Ten microliters of the MRSA solution (approximately $10^{4} \mathrm{CFU} / \mathrm{mL}$ ) was added in all wells (but not in well 11) and further incubated at $37^{\circ} \mathrm{C}$ in $\mathrm{O}_{2}$ atmosphere for 24 hours.

Next, to study the inhibitory effect of the nanoparticles, we inoculated the content $(5 \mu \mathrm{L})$ of each well in Petri dishes containing specific culture medium. The density of inoculum was standardized to give $10^{4} \mathrm{CFU}$ per spot on the agar. The MIC was considered the lowest concentration of the nanoparticles in which no bacterial growth was noticed (as judged by the naked eye), disregarding a single colony within the surface of the inoculated spot. Wells 11 and 12 were the positive (culture medium plus inoculum) and negative (culture medium plus antimicrobial) controls, respectively.

The same procedure was carried out for the GNPs in order to assess whether these nanoparticles did present any inhibitive effect on the MRSA culture.

\section{Viability measurements}

Following treatment, $1 \times 10^{4}$ microorganisms in MEM nonessential amino acid solution were pelleted by centrifugation
(14,000 RPM, 30 minutes). To evaluate MRSA viability after treatment, LIVE/DEAD BacLight Bacterial Viability test was performed in accordance with the manufacturer's instructions.

\section{Flow cytometry data analysis}

MRSA viability tests were assessed using a FACSCalibur (Becton Dickinson, San Jose, CA, USA) flow cytometer with green (FL1 channel $-530 \mathrm{~nm}$ ) and red (FL3 channel $>670$ $\mathrm{nm}$ ) fluorescence employed to distinguish between live and dead populations of microorganisms. Following administration of pre-GNPs, bacterial cultures were analyzed using LIVE/ DEAD BacLight kit. Suspensions of treated MRSA (GNPs/preGNPs) were analyzed by flow cytometry in accordance with the manufacturer's suggestions. The obtained histogram was further used to determine the percentage of live and dead bacteria using CellQuest ${ }^{\mathrm{TM}}$ Pro (BD Biosciences, San Jose, CA, USA) software. All analyzed variables were collected at low speed, in logarithmic mode (approximately $15 \mu \mathrm{L} \mathrm{min}^{-1}$ ), in order to maintain the counting level under 1,000 events per second. Characterization of bacterial populations was performed using Bacteria Counting Kit (Life Technologies) for flow cytometry. The kit includes the $\mathrm{SYTO}^{\circledR} \mathrm{BC}$ dye (green fluorescent dye; Life Technologies), a nucleic acid stain with high penetrance in Gram-negative bacteria. Signals from the fluorescently labeled bacteria where detected in the green fluorescence channel being displayed on a plot of forward scatter versus fluorescence, in accordance with the manufacturer protocol.

\section{Laser irradiation of GNPs}

GNPs (1 mL of GNP solution at various concentrations placed in a glass cuvette with approximately $1 \mathrm{~cm}^{2}$ bottom surface) were photoexcited for 10 minutes, using an $808 \mathrm{~nm}$ laser with variable output power (up to $2 \mathrm{~W} / \mathrm{cm}^{2}$ ). The laser diode was fixed above the surface, approximately $2 \mathrm{~cm}$, in a vertical position.

\section{MRSA-screen latex agglutination test}

The MRSA-screen latex agglutination test (MRSA-Screen; Denka Seiken Co, Ltd, Tokyo, Japan) - a qualitative slide latex agglutination test for the detection of penicillin-binding protein 2' (PBP2; also called PBP2a) present in isolates of $S$. aureus was performed according to the manufacturer's instructions. The test is extremely useful in identifying MRSA. A suspension of $5 \mu \mathrm{L} S$. aureus organisms suspended in alkaline extraction agent $(0.1 \mathrm{M} \mathrm{NaOH})$ was boiled for 3 minutes, and $50 \mu \mathrm{L}$ ( 1 drop) of extraction reagent $2\left(0.5 \mathrm{M} \mathrm{KH}_{2} \mathrm{PO}_{4}\right)$ was added. The mixture was centrifuged at $1,500 \times g$ for 5 minutes 
at room temperature, and $50 \mu \mathrm{L}$ of the supernatant was mixed with the latex reagent on a test card. As a negative control, $50 \mu \mathrm{L}$ of the supernatant was mixed with 1 drop $(25 \mu \mathrm{L})$ of negative-control latex. After the contents of the slide were mixed on a shaker, agglutination within 3 minutes indicates the presumptive presence of_PBP $2 .^{31}$

\section{Statistical data analysis}

All parameters were expressed as the mean and standard error of the mean. Kolmogorov-Smirnov test was used to assess non-normal data. The Wilcoxon signed-rank test was used to compare similar parameters within different groups. In all cases, $\alpha$ error level was under 0.05. Data were processed using Excel ${ }^{\circledR}$ (Microsoft Corporation, Redmond, WA, USA) application, as well as SPSS Statistics (IBM Corporation, Armonk, NY, USA), version 17.0 packages.

\section{Results}

\section{Characterization of GNPs}

The main goal of this investigation was to study the interaction between MRSA bacteria and laser pre-GNPs (Figure 1). As seen in Figure 1A, there was an average diameter of $27 \mathrm{~nm}$ of the synthesized GNPs, as suggested by the Zetasizer DLS system. To monitor changes in absorption and scattering peaks following irradiation, we further displayed the UVvisable absorption peak shifts against laser power. As seen in Figure 2, the peak wavelength shifted to longer wavelengths together with the increase in laser power, a characteristic that has also been described by other authors. ${ }^{32}$

Prior to laser irradiation, the specific SPR adsorption peak of the GNPs was at $530 \mathrm{~nm}$ (Figure 2, black spectra). When the laser irradiation was initiated, the scattering spectrum of GNPs exhibited a peak shifting to longer wavelengths, and by $1 \mathrm{~W} / \mathrm{cm}^{2}$, an approximately $13 \mathrm{~nm}$ total peak shift

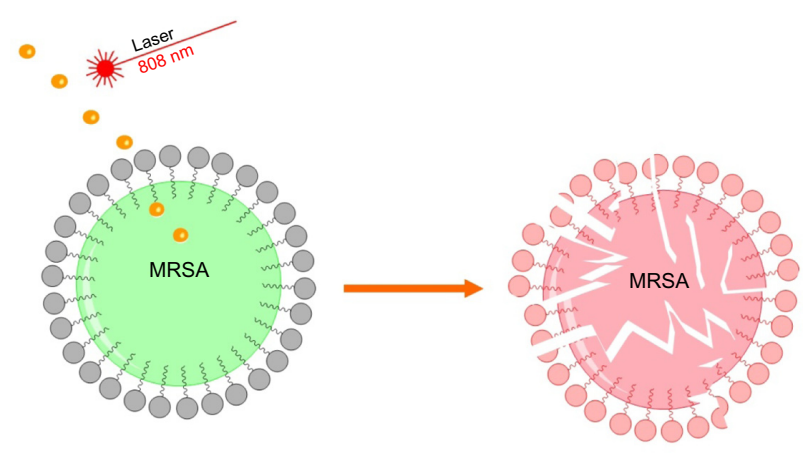

Figure I Schematic illustration of the proposed antimicrobial nanotherapy against MRSA, using laser pre-irradiated gold nanoparticles.

Abbreviation: MRSA, methicillin-resistant Staphylococcus aureus. was noticed, strongly suggesting an induction of SPR phenomenon. Similar to other authors, we noted that the peak shift was caused by laser power instead of exposure time. It is well known that GNPs can induce extreme local heating on a timescale of up to several nanoseconds. ${ }^{32}$

At this point, we showed that laser photoexcitation of conduction electrons induced their oscillation and consequent non-radiative relaxation through electron-phonon and subsequent phonon-phonon coupling mechanisms. We further assessed the interaction of these photoexcited nanoparticles (in a non-radiative relaxation state) with the MRSA bacteria.

\section{Phase contrast and fluorescence microscopy}

After incubation with pre-GNPs, phase contrast microscopy was performed to demonstrate the interaction of MRSA with pre-GNPs following treatment. As seen in Figure $2 \mathrm{C}$ and $\mathrm{D}$, dark and optically dense signals that associate with a refringent signal in phase contrast examination (strongly suggesting GNPs) were noticed in these bacteria. These observations strongly suggest intracellular aggregates of GNPs pre-treated with laser radiation (Figure 2D). We were able to identify these aggregates inside MRSA bacteria that had undergone treatment with GNP solution alone, but these aggregates were significantly fewer and barely visible in contrast phase microscopy (Figure 2C).

To confirm the intensity of MRSA destruction following treatment, we further used immunofluorescence microscopy (LIVE/DEAD BacLight kit). In accordance with flow cytometric quantification, as seen in Figure 3, bacteria treated with pre-GNPs revealed significantly increased red fluorescence, suggesting higher necrosis rates in MRSA culture compared with bacteria treated with GNPs alone (chi-square, $P<0.05$ ).

A moderate to high antimicrobial activity against the $S$. aureus microorganism was found for plasmonic activated GNPs (Table 1). For these nanoparticles, inhibition of microorganism growth corresponded to an MIC value of $6.25 \%$ of the concentration of the pre-GNPs solution. An unfavorable MIC of $25 \%$ was obtained for the solution containing only GNPs.

\section{Flow cytometry}

Antibacterial properties of nanoparticles are a consequence of changes in local electronic structures on their surfaces. As seen in Figures 4 and 5, following GNP therapy as described 

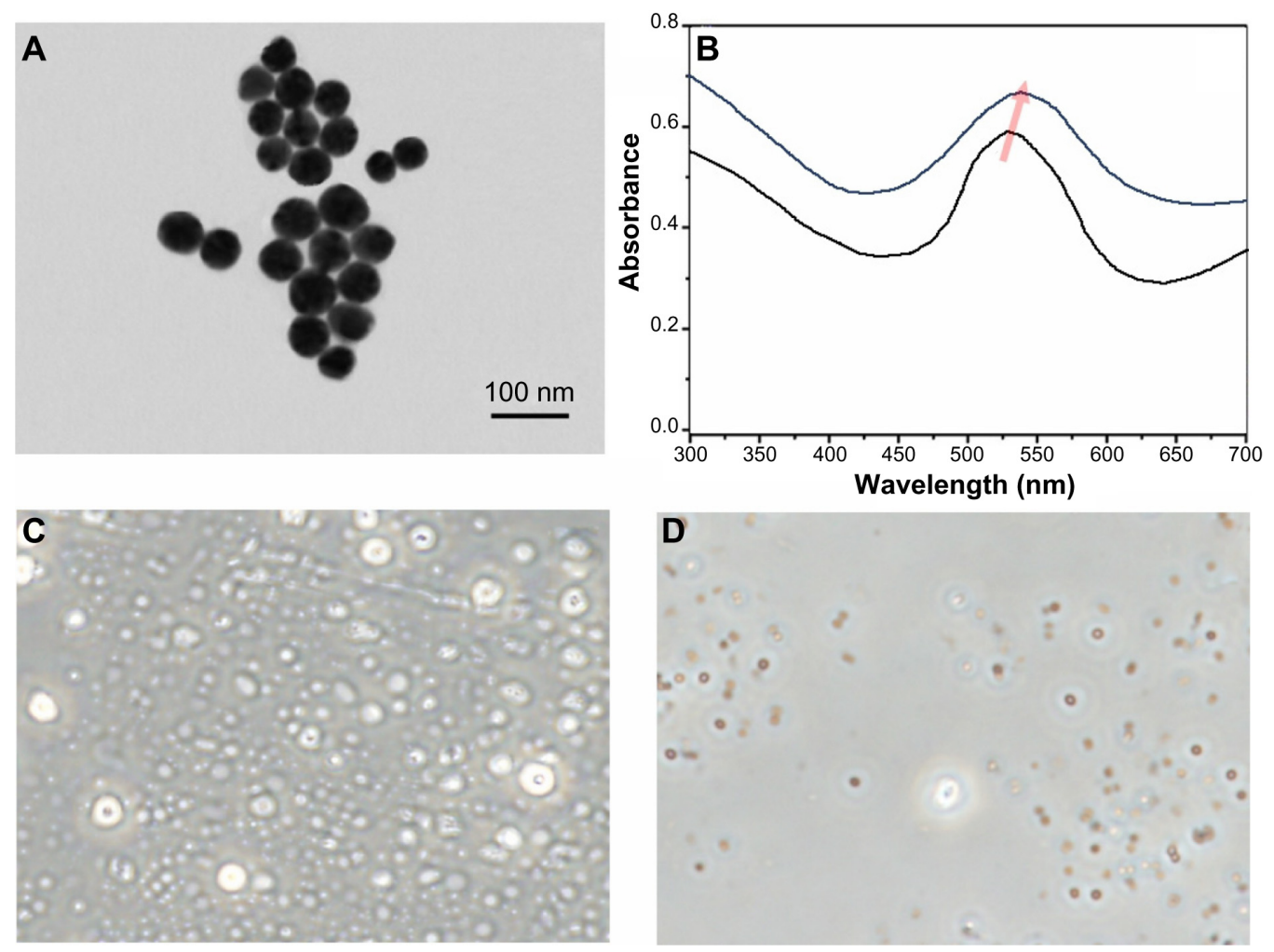

Figure 2 (A) Transmission electron micrographs of the GNPs used for the experiments. The scale bar represents $100 \mathrm{~nm}$. (B) Ultraviolet-visible relationship between peak wavelength and laser power in the pre-GNPs (black spectra $0.5 \mathrm{~W} / \mathrm{cm}^{2}$; blue spectra I W/cm²). (C) Phase contrast microscopy of MRSA treated with GNPs alone (5 mg/L). (D) Phase contrast microscopy of MRSA treated with pre-GNPs (5 mg/L).

Abbreviations: GNP, gold nanoparticle; MRSA, methicillin-resistant Staphylococcus aureus.

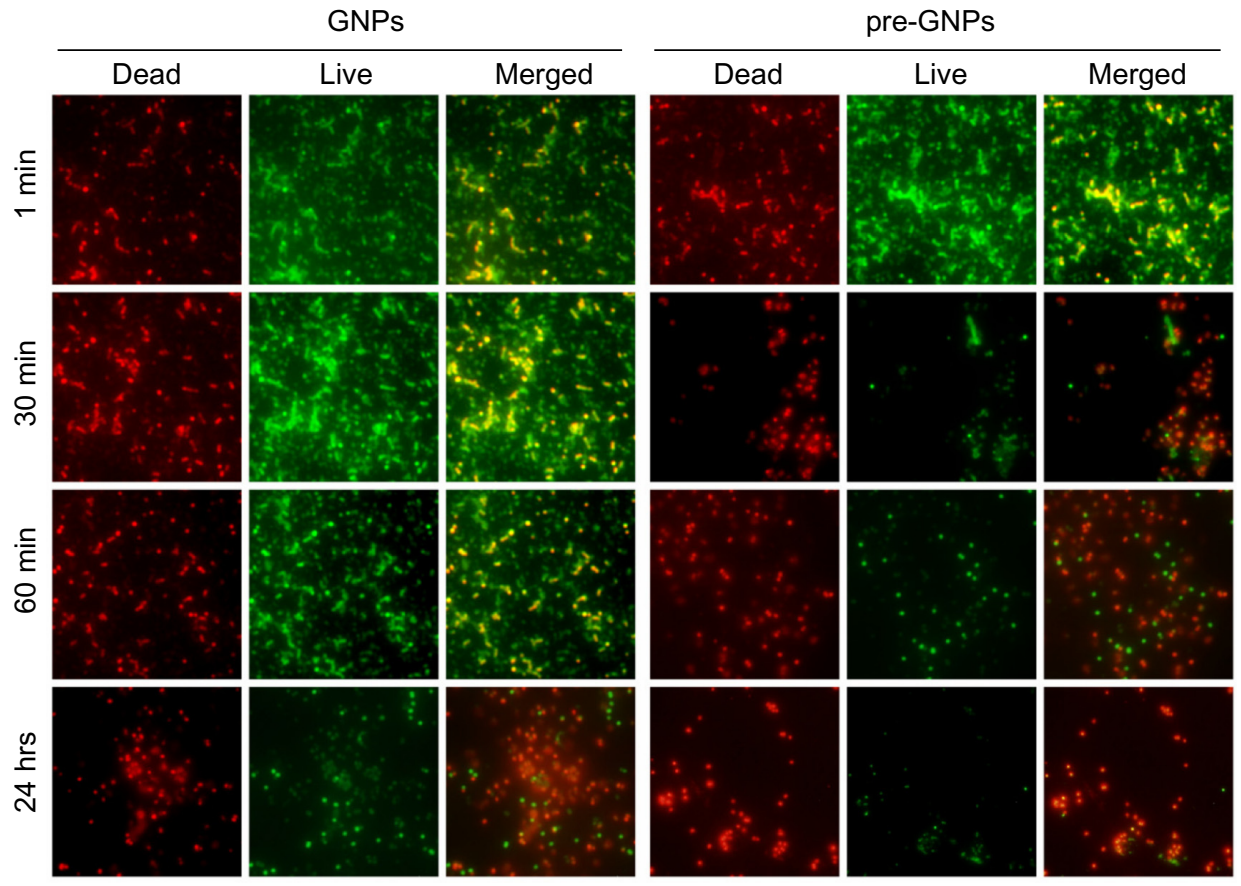

Figure 3 Immunofluorescence microscopy (LIVE/DEAD ${ }^{\circledR}$ BacLight ${ }^{T M}$ Bacterial Viability Kit; Life Technologies, Carlsbad, CA, USA) of the bacterial population following treatment with $5 \mathrm{mg} / \mathrm{L} \mathrm{GNPs} /$ pre-GNPs at various incubation times (red, dead bacteria; green, live bacteria). The merged images represent the superposition of dead and live bacteria.

Abbreviation: GNP, gold nanoparticle. 
Table I Minimum inhibitory concentration of GNPs against MRSA

\begin{tabular}{llll}
\hline Well & Percentage & GNPs & Pre-GNPs \\
\hline 1 & 100 & - & - \\
2 & 50 & - & - \\
3 & 25 & - & - \\
4 & 12.5 & + & - \\
5 & 6.25 & + & - \\
6 & 3.13 & + & + \\
7 & 1.56 & + & + \\
8 & 0.78 & + & + \\
9 & 0.39 & + & + \\
10 & 0.20 & + & + \\
11 & 0.10 & - & - \\
12 & - & + & + \\
\hline
\end{tabular}

Notes: +, growth of bacteria; -, no growth of bacteria; well II, negative control; well 12, positive control.

Abbreviations: GNP, gold nanoparticle; MRSA, methicillin-resistant Staphylococcus aureus.

above, the degree of MRSA destruction ranged from $28.4 \%$ (for $1 \mathrm{mg} / \mathrm{L}$ ) to $72.6 \%$ (for $50 \mathrm{mg} / \mathrm{L}$ ) at 60 seconds $(P<0.001$ ), while at 30 minutes, the percentage of dead bacteria increased from $45.2 \%(1 \mathrm{mg} / \mathrm{L})$ to $88.2 \%(50 \mathrm{mg} / \mathrm{L}), P<0.001$.

A

GNP
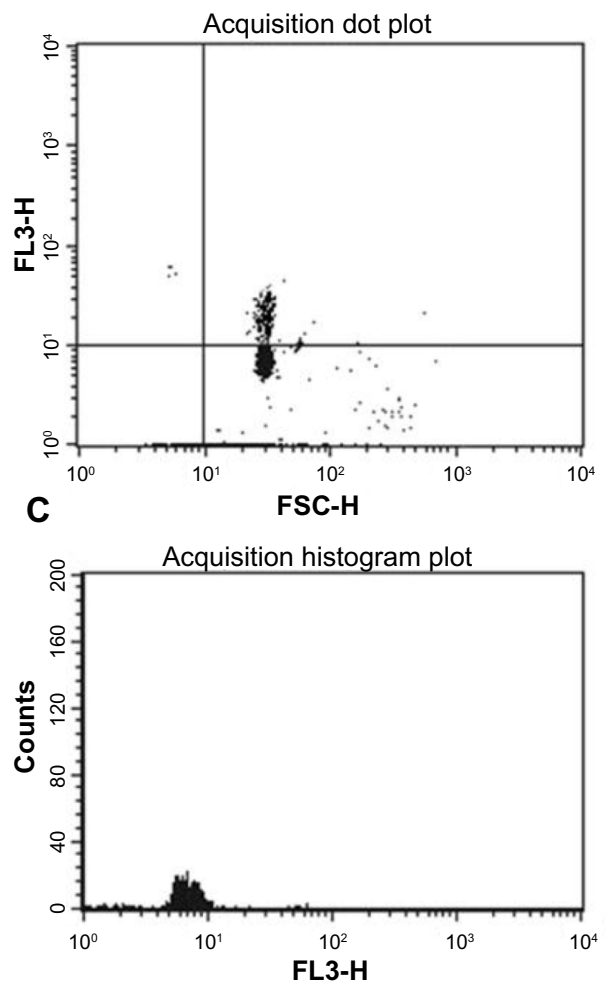

MRSA treated with GNPs alone (control) for 60 seconds and 30 minutes at concentrations ranging from 1 to $50 \mathrm{mg} / \mathrm{L}$ resulted in significantly lower rates of necrosis $(8.3 \%-39.6 \%$ for 60 seconds, $9.8 \%-51.3 \%$ for 30 minutes; $P<0.001)$. The optimum necrotic impact of MRSA induced by pre-GNPs was achieved following treatment with pre-GNPs at $5 \mathrm{mg} / \mathrm{L}$ for 30 minutes (pre-GNPs/GNPs: 53.3\%/16.2\% at 30 minutes). Following an incubation period of 3-5 hours, the percentage of MRSA destruction among the two GNP solutions in low concentrations $(<20 \mathrm{mg} / \mathrm{L})$ was of marginal statistical significance $(P=0.081)$. An effect on bacteria lysis was observed in the two treated MRSA cultures, associated with elevated concentrations, but the difference between them was not significant $(P=0.316$ for $20 \mathrm{mg} / \mathrm{L} ; P=0.438$ for $50 \mathrm{mg} / \mathrm{L}$ ).

\section{Discussion}

From a clinical perspective, a revolutionary antimicrobial therapy for serious infections caused by MRSA would be represented by agents that cause physical damage to the bacteria. ${ }^{33-36}$ In this context, one of the most promising and

B
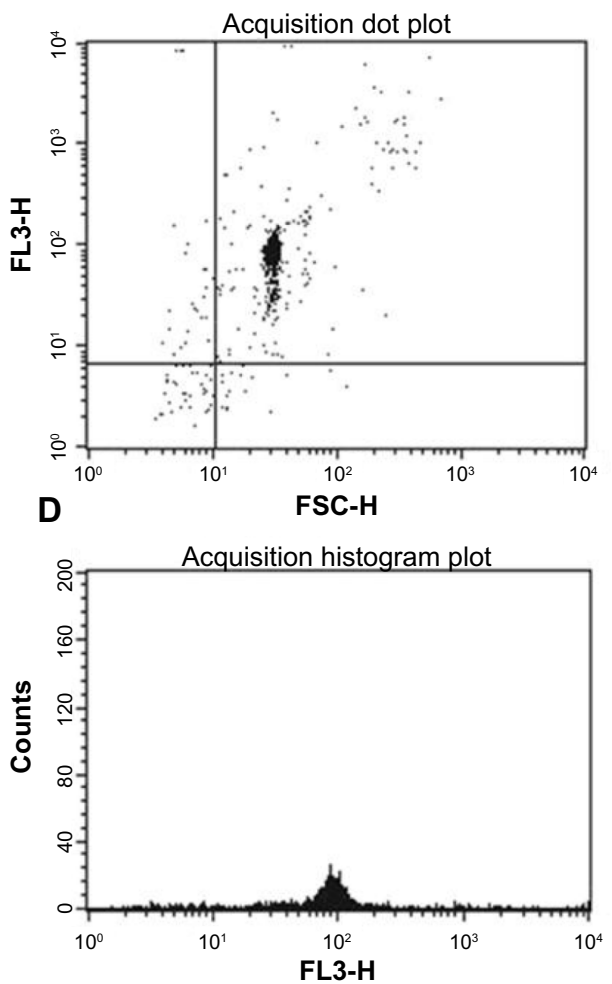

Figure 4 Flowcytometric quantification of bacterial lysis in MRSA microorganism following pre-GNPs treatment.

Notes: (A and B) representative FACS dot plots (red fluorescence[FL3] versus forward scatter[FSC]) gated on MRSA population are shown. (B) show increased red fluorescence (FL3) suggesting high viability loss after LASER irradiation following incubation for I hour with $5 \mathrm{mg} / \mathrm{L}$ pre-GNPs compared with GNPs only (A). (C and D) display histogram representation of red fluorescence, (dead bacteria) in LASER treated MRSA population following GNPs treatment (C) or pre-GNPs (D). Abbreviations: FACS, fluorescence-activated cell sorting; GNPs, gold nanoparticles; MRSA, methicillin-resistant Staphylococcus aureus. 


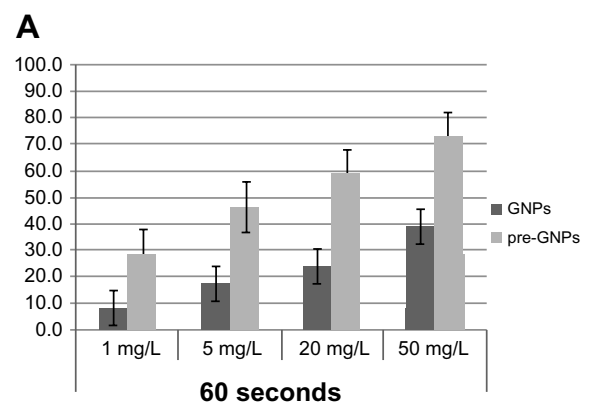

\section{B}

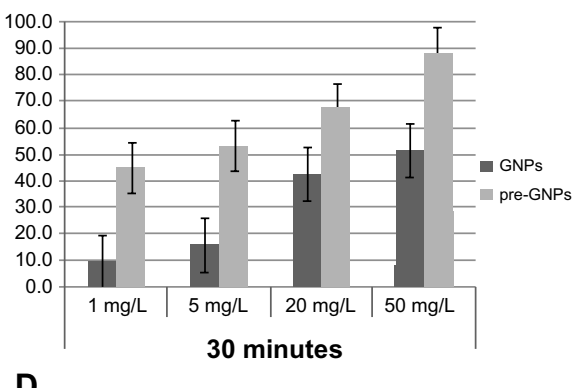

D

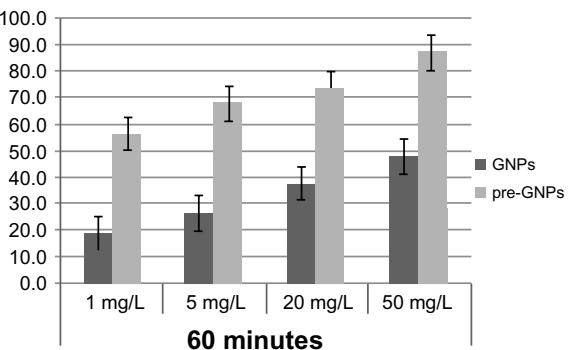

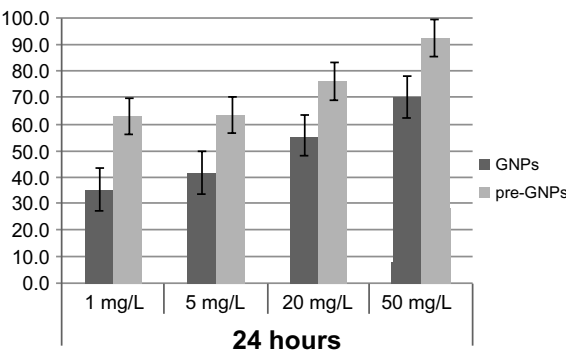

24 hours

Figure 5 Results of experimental seriate exposure of MRSA to gold nanoparticles in different concentrations.

Notes: Bars represent median dead bacteria percent as calculated by flow cytometry. Error bars represent range of dead bacteria percent. MRSA treated with GNPs only (control) for 60 seconds (A) and 30 minutes (B) at concentrations ranging from I mg/L to $50 \mathrm{mg} / \mathrm{L}$ resulted in significantly lower necrotic rates. Following treatment for 60 minutes (C) the percentages of MRSA necrosis among the two GNPs solutions in low concentrations $(<20 \mathrm{mg} / \mathrm{L})$ was statistically significant. Treatment with high concentration of GNPs for a long period of time (24 hours) (D) did not reveal any significance in terms of necrotic percentage among the two MRSA cultures (GNPs and pre-GNPs). Abbreviations: GNP, gold nanoparticle; MRSA, methicillin-resistant Staphylococcus aureus.

innovative approaches to treat MRSA infections would be to deliver highly efficient active biomolecules to the site of infection and selectively target bacteria over other cells. The therapeutic efficacy of the drug should also be easily ascertained. Since the development of resistance is expected to continue in the coming years, there is a dire need for the development of novel antibacterial agents that would direct the active molecule to the infection site. These alternative methods against MRSA, towards which no resistance can develop, would remove and inhibit those organisms relevant to infection and would be useful against all virulent strains.

Several different mechanisms of antibiotic resistance may exist. Thus, changes of the molecular or metabolic epitopes onto the surface of microorganism, chromosomal mutations or enzymatic degradation of the antibiotic, and active transportation of the antibiotic out of the cell or periplasm are the most common ways of development of antibiotic resistance in bacteria. ${ }^{37}$

To overcome the antibiotic resistance, ${ }^{38-40}$ several authors ${ }^{41}$ developed highly stable water soluble vancomycin-covered GNPs as polyvalent inhibitors, proving their effectiveness against vancomycin-resistant strains and Gram-negative bacteria, including MRSA. In this experiment, GNPs (4-5 nm, obtained in toluene) were conjugated with bis(vancomycin) cystamide (in $\mathrm{H}_{2} \mathrm{O}$ ) to generate $\mathrm{Au}-\mathrm{S}$ bonds that link vancomycin to gold. Finally, gold-vancomycin nanoparticles were dissolved in aqueous solution and further removed from the organic phase.

Rosemary et $\mathrm{al}^{42-44}$ developed a complex made of ciprofloxacin and gold nanoshells that was further tested against Escherichia coli DH5R and Lactococcus lactis MG1363 using the agar dilution method, results being compared to those of free ciprofloxacin. Studies of hydrophobicity on ciprofloxacin@ $\mathrm{SiO}_{2}$ showed a different penetration pathway compared to the free drug. Fluorescein-isothiocyanate@ $\mathrm{SiO}_{2}$ helped perform nanoshell/bacteria interaction studies. The fluorescence image suggested shell internalization into the microorganism. Transmission electron microscopy was further performed on ciprofloxacin@ $\mathrm{SiO}_{2}$-treated E. coli to confirm any possible changes in morphology following treatment.

Several authors ${ }^{45}$ found that GNPs mainly exert their antibacterial action in two ways. The first one occurs by changing membrane potential and inhibiting adenine triphosphate (ATP) synthase activities to lower the level of ATP, activating metabolic pathways that generate anabolic reactions. The other occurs by inhibiting the subunit of ribosome for tRNA (transfer ribonucleic acid) binding, generating a blockade of the vital reactions in bacteria. They also proved that GNPs enhance chemotaxis in the earlyphase reaction and that bactericidal GNPs did not induce any ROS (reactive oxygen species)-related process. ${ }^{46}$ 
To our knowledge, our discovery represents an initial report where active plasmonic GNPs exert antimicrobial effects on MRSA bacteria. We have previously demonstrated the therapeutic impact of plasmon-active GNPs on pancreatic cancer cells. $^{47}$

From a surgical point of view, the imaging diagnosis (by computed tomography scan or ultrasound) of an abscess and its external drainage followed by the administration of the pre-activated GNPs, as well as the external laser irradiation for complete eradication of MRSA bacteria biofilm (which is responsible for the recurrence of infections), could all be safely achieved in patients by means of laparoscopic/minimal invasive surgery. Nevertheless, further investigation is required for the careful assessment of unexpected toxicities and biological interactions of bio-nanomaterials within biological systems. ${ }^{8,48-52}$

\section{Conclusion}

We showed here that phonon-phonon interactions following laser photoexcitation of GNPs exhibit increased MRSA necrosis rates at low concentrations and short incubation times compared with MRSA treated with GNPs alone. These unique data may offer new perspectives in the field of nanomediated antibiotherapy.

\section{Acknowledgment}

This work was funded by the Romanian Ministry of Education and Research (PN-II-PT-PCCA-2011-3.1-1586, PNII-RU-TE-2011-3-0251; PN-II-PT-PCCA-2011-3.1-1551; PN-II-PT-PCCA-2011-3.2-1289).

\section{Disclosure}

The authors report no conflicts of interest in this work.

\section{References}

1. Alanis AJ. Resistance to antibiotics: are we in the post-antibiotic era? Arch Med Res. 2005;36(6):697-705.

2. Cosgrove SE, Qi Y, Kaye KS, Harbarth S, Karchmer AW, Carmeli Y. The impact of methicillin resistance in Staphylococcus aureus bacteremia on patient outcomes: mortality, length of stay, and hospital charges. Infect Control Hosp Epidemiol. 2005;26(2):166-174.

3. Gardete S, Kim C, Hartmann BM, et al. Genetic pathway in acquisition and loss of vancomycin resistance in a methicillin resistant Staphylococcus aureus (MRSA) strain of clonal type USA300. PLoS Pathog. 2012;8(2):e1002505.

4. Köck R, Becker K, Cookson B, et al. Methicillin-resistant Staphylococcus aureus (MRSA): burden of disease and control challenges in Europe. Euro Surveill. 2010;15(41):19688.

5. Ke W, Huang SS, Hudson LO, et al. Patient sharing and population genetic structure of methicillin-resistant Staphylococcus aureus. Proc Natl Acad Sci U SA. 2012;109(17):6763-6768.

6. Harris SR, Feil EJ, Holden MT, et al. Evolution of MRSA during hospital transmission and intercontinental spread. Science. 2010; 327(5964):469-474.
7. dos Santos CA, Jozala AF, Pessoa A Jr, Seckler MM. Antimicrobial effectiveness of silver nanoparticles co-stabilized by the bioactive copolymer pluronic F68. J Nanobiotechnology. 2012;10(1):43.

8. Iancu C, Ilie IR, Georgescu CE, et al. Applications of nanomaterials in cell stem therapies and the onset of nanomedicine. Particul Sci Technol. 2009;27(6):562-574.

9. Iancu C, Mocan L, Bele C, et al. Enhanced laser thermal ablation for the in vitro treatment of liver cancer by specific delivery of multiwalled carbon nanotubes functionalized with human serum albumin. Int $J$ Nanomedicine. 2011;6:129-141.

10. Peer D, Karp JM, Hong S, Farokhzad OC, Margalit R, Langer R. Nanocarriers as an emerging platform for cancer therapy. Nature nanotechnology. 2007;2(12):751-760.

11. Liu Z, Davis C, Cai W, He L, Chen X, Dai H. Circulation and long-term fate of functionalized, biocompatible single-walled carbon nanotubes in mice probed by Raman spectroscopy. Proc Natl Acad Sci U S A. 2008;105(5):1410.

12. Lin M, Pei H, Yang F, Fan C, Zuo X. Applications of gold nanoparticles in the detection and identification of infectious diseases and biothreats. Adv Mater. 2013;25(25):3490-3496.

13. Mieszawska AJ, Mulder WJ, Fayad ZA, Cormode DP. Multifunctional gold nanoparticles for diagnosis and therapy of disease. Mol Pharm. 2013;10(3):831-847.

14. Dreaden EC, Alkilany AM, Huang X, Murphy CJ, El-Sayed MA. The golden age: gold nanoparticles for biomedicine. Chem Soc Rev. 2012;41(7):2740-2779.

15. Ilie I, Ilie R. Models of regeneration of beta cells in vivo. Available from http://researchpub.org/journal/bmbn/number/vol1-no1/vol1-no1-3.pdf. Accessed March 3, 2014.

16. Mocan L. Drug delivery applications of gold nanoparticles. Available from http://researchpub.org/journal/bmbn/number/vol1-no1/vol1no1-1.pdf. Accessed March 3, 2014.

17. Eustis S, El-Sayed MA. Why gold nanoparticles are more precious than pretty gold: noble metal surface plasmon resonance and its enhancement of the radiative and nonradiative properties of nanocrystals of different shapes. Chem Soc Rev. 2005;35(3):209-217.

18. Huang X, Jain PK, El-Sayed IH, El-Sayed MA. Plasmonic photothermal therapy (PPTT) using gold nanoparticles. Lasers Med Sci. 2008;23(3):217-228.

19. Guillot N, Shen H, Fremaux B, et al. Surface enhanced Raman scattering optimization of gold nanocylinder arrays: influence of the localized surface plasmon resonance and excitation wavelength. Appl Phys Lett. 2010;97:023113.

20. Link S, El-Sayed MA. Shape and size dependence of radiative, nonradiative and photothermal properties of gold nanocrystals. Int Rev Phys Chem. 2000;19(3):409-453.

21. Dykman L, Khlebtsov N. Gold nanoparticles in biomedical applications: recent advances and perspectives. Chem Soc Rev. 2012;41(6): 2256-2282.

22. Jain S, Hirst D, O'sullivan J. Gold nanoparticles as novel agents for cancer therapy. Br J Radiol. 2012;85(1010):101-113.

23. Llevot A, Astruc D. Applications of vectorized gold nanoparticles to the diagnosis and therapy of cancer. Chem Soc Rev. 2012;41(1): 242-257.

24. Yeh Y, Creran B, Rotello VM. Gold nanoparticles: preparation, properties, and applications in bionanotechnology. Nanoscale. 2012;4(6): 1871-1880.

25. Dykman L, Khlebtsov N. Gold nanoparticles in biology and medicine: recent advances and prospects. Acta Naturae. 2011;3(2):34-55.

26. Gu T, Ye T, Simon JD, Whitesell JK, Fox MA. Subpicosecond transient dynamics in gold nanoparticles encapsulated by a fluorophore-terminated monolayer. J Phys Chem B. 2003;107(8):1765-1771.

27. Mukherjee P, Bhattacharya R, Bone N, et al. Potential therapeutic application of gold nanoparticles in B-chronic lymphocytic leukemia (BCLL): enhancing apoptosis. J Nanobiotechnology. 2007;5:4.

28. Haiss W, Thanh NT, Aveyard J, Fernig DG. Determination of size and concentration of gold nanoparticles from UV-vis spectra. Anal Chem. 2007;79(11):4215-4221. 
29. Cho SP, Bratescu MA, Saito N, Takai O. Microstructural characterization of gold nanoparticles synthesized by solution plasma processing. Nanotechnology. 2011;22(45):455701.

30. Mazzola PG, Jozala AF, Novaes LCL, Moriel P, Penna TCV. Minimal inhibitory concentration (MIC) determination of disinfectant and/or sterilizing agents. Braz J Pharm Sci. 2009;45(2):241-248.

31. van Leeuwen WB, van Pelt C, Luijendijk A, Verbrugh HA, Goessens WH. Rapid detection of methicillin resistance in Staphylococcus aureus isolates by the MRSA-Screen latex agglutination test. J Clin Microbiol. 1999;37(9):3029-3030.

32. Honda M, Saito Y, Smith NI, Fujita K, Kawata S. Nanoscale heating of laser irradiated single gold nanoparticles in liquid. Optics Express. 2011;19(13):12375-12383.

33. Sharma A, Kumar Arya D, Dua M, Chhatwal GS, Johri AK. Nano-technology for targeted drug delivery to combat antibiotic resistance. Expert Opin Drug Deliv. 2012;9(11):1325-1332.

34. Burygin G, Khlebtsov B, Shantrokha A, Dykman L, Bogatyrev V, Khlebtsov N. On the enhanced antibacterial activity of antibiotics mixed with gold nanoparticles. Nanoscale Res Lett. 2009;4(8): 794-801.

35. Schmidt MA. Beyond Antibiotics: Strategies for Living in a World of Emerging Infections and Antibiotic-Resistant Bacteria. Berkeley, CA: North Atlantic Books; 2009.

36. Huh AJ, Kwon YJ. "Nanoantibiotics": a new paradigm for treating infectious diseases using nanomaterials in the antibiotics resistant era. J Control Release. 2011;156(2):128-145.

37. Giedraitienè A, Vitkauskienè A, Naginienè R, Pavilonis A. Antibiotic resistance mechanisms of clinically important bacteria. Medicina (Kaunas). 2011;47(3):137-146.

38. Choi CHJ, Alabi CA, Webster P, Davis ME. Mechanism of active targeting in solid tumors with transferrin-containing gold nanoparticles. Proc Natl Acad Sci U S A. 2010;107(3):1235-1240.

39. Yang P, Sun X, Chiu J, Sun H, He Q. Transferrin-mediated gold nanoparticle cellular uptake. Bioconjug Chem. 2005;16(3): 494-496.

40. Chithrani BD, Chan WC. Elucidating the mechanism of cellular uptake and removal of protein-coated gold nanoparticles of different sizes and shapes. Nano Lett. 2007;7(6):1542-1550.

41. $\mathrm{Gu} \mathrm{H}, \mathrm{Xu} \mathrm{K}, \mathrm{Xu} \mathrm{C}, \mathrm{Xu}$ B. Biofunctional magnetic nanoparticles for protein separation and pathogen detection. Chem Commun (Camb). 2006;(9): 941-949.
42. Rosemary M, MacLaren I, Pradeep T. Investigations of the antibacterial properties of ciprofloxacin@SiO2. Langmuir. 2006;22(24): 10125-10129.

43. Selvaraj V, Alagar M. Analytical detection and biological assay of antileukemic drug 5-fluorouracil using gold nanoparticles as probe. Int J Pharm. 2007;337(1):275-281.

44. Rosemary M, Suryanarayanan V, Ganapatireddy P, Maclaren I, Baskaran S, Pradeep T. Ciprofloxacin@ $\mathrm{SiO}_{2}$ : fluorescent nanobubbles. Journal of Chemical Sciences. 2003;115(5-6):703-709.

45. Cui Y, Zhao Y, Tian Y, Zhang W, Lü X, Jiang X. The molecular mechanism of action of bactericidal gold nanoparticles on Escherichia coli. Biomaterials. 2012;33(7):2327-2333.

46. Martinez-Castanon GA, Nino-Martinez N, Martinez-Gutierrez F, Martinez-Mendoza JR, Ruiz F. Synthesis and antibacterial activity of silver nanoparticles with different sizes. J Nanopart Res. 2008;10(8): 1343-1348.

47. Mocan L, Ilie I, Tabaran FA, et al. Surface plasmon resonance-induced photoactivation of gold nanoparticles as mitochondria-targeted therapeutic agents for pancreatic cancer. Expert Opin Ther Targets. 2013;17(12):1383-1393.

48. Mocan T. Hemolysis as expression of nanoparticles-induced cytotoxicity in red blood cells. Available from http://researchpub.org/ journal/bmbn/number/vol1-no1/vol1-no1-2.pdf. Accessed March 3, 2014.

49. Mocan T, Clichici S, Biris AR, et al. Dynamic effects over plasma redox balance following subcutaneous injection of single walled carbon nanotubes functionalized with single strand DNA. Dig J Nanomat Bios. 2011;6(3):1207-1214.

50. Li JJ, Hartono D, Ong CN, Bay BH, Yung LY. Autophagy and oxidative stress associated with gold nanoparticles. Biomaterials. 2010;31(23): 5996-6003.

51. Boisselier E, Astruc D. Gold nanoparticles in nanomedicine: preparations, imaging, diagnostics, therapies and toxicity. Chem Soc Rev. 2009;38(6):1759-1782.

52. Male KB, Lachance B, Hrapovic S, Sunahara G, Luong JHT. Assessment of cytotoxicity of quantum dots and gold nanoparticles using cell-based impedance spectroscopy. Anal Chem. 2008;80(14):5487-5493.
International Journal of Nanomedicine

\section{Publish your work in this journal}

The International Journal of Nanomedicine is an international, peerreviewed journal focusing on the application of nanotechnology in diagnostics, therapeutics, and drug delivery systems throughout the biomedical field. This journal is indexed on PubMed Central,

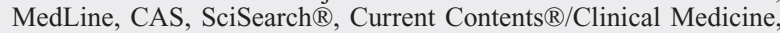

\section{Dovepress}

Journal Citation Reports/Science Edition, EMBase, Scopus and the Elsevier Bibliographic databases. The manuscript management system is completely online and includes a very quick and fair peer-review system, which is all easy to use. Visit http://www.dovepress.com/ testimonials.php to read real quotes from published authors. 